\title{
Stability and nonlinear adjustment of vortices in Keplerian flows
}

\author{
G. Bodo ${ }^{1}$, A. Tevzadze ${ }^{2}$, G. Chagelishvili ${ }^{2}$, A. Mignone ${ }^{1,3}$, P. Rossi $^{1}$, and A. Ferrari ${ }^{3}$ \\ 1 INAF Osservatorio Astronomico di Torino, Strada dell'Osservatorio 20, 10025 Pino Torinese, Italy \\ e-mail: bodo@to.astro.it \\ 2 E. Kharadze Georgian National Astrophysical Observatory,, 2a Kazbegi Ave. Tbilisi 0160, Georgia \\ 3 Dipartimento di Fisica Generale dell'Università di Torino, via Pietro Giuria 1, 10125 Torino, Italy
}

Received 23 April 2007 / Accepted 27 August 2007

\begin{abstract}
Aims. We investigate the stability, nonlinear development and equilibrium structure of vortices in a background shearing Keplerian flow

Methods. We make use of high-resolution global two-dimensional compressible hydrodynamic simulations. We introduce the concept of nonlinear adjustment to describe the transition of unbalanced vortical fields to a long-lived configuration.

Results. We discuss the conditions under which vortical perturbations evolve into long-lived persistent structures and we describe the properties of these equilibrium vortices. The properties of equilibrium vortices appear to be independent from the initial conditions and depend only on the local disk parameters. In particular we find that the ratio of the vortex size to the local disk scale height increases with the decrease of the sound speed, reaching values well above the unity. The process of spiral density wave generation by the vortex, discussed in our previous work, appear to maintain its efficiency also at nonlinear amplitudes and we observe the formation of spiral shocks attached to the vortex. The shocks may have important consequences on the long term vortex evolution and possibly on the global disk dynamics.

Conclusions. Our study strengthens the arguments in favor of anticyclonic vortices as the candidates for the promotion of planetary formation. Hydrodynamic shocks that are an intrinsic property of persistent vortices in compressible Keplerian flows are an important contributor to the overall balance. These shocks support vortices against viscous dissipation by generating local potential vorticity and should be responsible for the eventual fate of the persistent anticyclonic vortices. Numerical codes have be able to resolve shock waves to describe the vortex dynamics correctly.
\end{abstract}

Key words. accretion, accretion disks - planets and satellites: formation - hydrodynamics - methods: numerical

\section{Introduction}

Recent advances in the understanding of vortex behavior in differentially rotating flows are mainly associated with the study of protoplanetary disk dynamics, since von Weiszacker (1944) and Adams \& Watkins (1995), who suggested that vortices can promote the formation of planetesimals. Indeed, it has been shown that vortices, if sustained long enough, lead to particle aggregation in their core (see e.g. Chavanis 2000; de la Fuente Marcos \& Barge 2001; Johansen et al. 2004; Klahr \& Bodenheimer 2006) and to the formation of protoplanets.

The vortex scenario for planetary formation encounters an apparent obstacle: any structure in a Keplerian disk is subject to a strong shearing that may eventually lead to its decay. The only mechanism for sustaining a stable vortex in such flows is nonlinearity. Hence, vortices that may start the process of planetary formation should exceed a critical threshold in their amplitude. Direct numerical simulations are therefore an important tool in these studies.

Previous studies on vortex stability, performed mainly by numerical simulations (see Barge \& Sommeria 1995; Bracco et al. 1999; Godon \& Livio 1999b,a; Davis et al. 2000; Davis 2002; Klahr \& Bodenheimer 2003; Klahr 2004; Barranco \& Marcus 2005; Umurhan \& Regev 2004), have shown that only anticyclonic vortices can be nonlinearly stable in Keplerian flows and can promote dust trapping and the creation of protoplanets. Similar indications come from hydrodynamical simulations of accretion disks with initially imposed random vorticity fields, where again only anticyclonic vortices survive (Shen et al. 2006; Johnson \& Gammie 2005).

Although different numerical simulations have already shown that anticyclonic vortices can survive in compressible Keplerian disks, most of the stable structures are observed on scales smaller than the scale height of the disk. This calls for three-dimensional investigations before solid conclusions can be drawn. On the other hand, Barranco \& Marcus (2005) have found indications of a 3D vortex instability and have shown that only off mid-plane vortices with a very specific vertical structure are stable in 3D. The preferred location for dust capture and planetesimals are, however, the high density regions in the midplane of the disk, where vortices are unstable. A solution to this problem could be the existence of vortices with a horizontal extent larger than the disk thickness. Those should in fact behave as 2D structures therefore being stable and promote mass accumulation. However, presently, there are doubts on the possibility of the existence of such vortices (see Johnson \& Gammie 2005; Barranco \& Marcus 2005). They are thought to decay to a smaller size, of the order of the disk scale height, due to the supersonic velocity circulation.

In the present paper, we study the nonlinear dynamics of relatively large scale (exceeding the disk half thickness) vortices in keplerian flows by global compressible simulations. In this situation, compressibility is an important factor that cannot be neglected because the Keplerian profile, setting a strong radial velocity shear, couples necessarily vortex and wave mode 
perturbation (see Bodo et al. 2005). Our aim is to study in detail equilibrium structure of nonlinear long lived vortices. In our study we assume the initial vortical perturbation as given and we investigate how it evolves into an equilibrium configuration and the properties of this configuration. It is not our aim here to study how such large amplitude perturbation was formed. Recent studies (see e.g. Klahr \& Bodenheimer 2003; Petersen et al. 2007) have pointed out how baroclinic effects can lead to a growth of vortical perturbations, but that a radial temperature variation is required. However, here, for studying the vortex structure, we assume, for simplicity, a disk with initially uniform temperature. The adequacy of this approximation must be tested through future studies. We will focus our analysis on the possible existence of vortices with horizontal extent larger than the disk scale height. An important process for the vortex dynamics in compressible flows is the linear generation of spiral density waves, described in Bodo et al. (2005). In the present paper we further investigate this process by verifying its efficiency during the nonlinear stages and by analyzing its relevance for the vortex structure. In this respect, an interesting point is that equilibrium vortex structures work as a mill that processes the shear flow energy into coherent wave emission. The nonlinear development of spiral-density waves leads to the formation of spiral shocks with a steady pattern. These shocks may increase the stability of anticyclonic vortices by slowing down their decay and may have also global effect on the disk.

In Sect. 2 we describe the numerical code, setup and initial conditions used in the simulations. In Sect. 3 we present the results of our numerical simulations and describe the nonlinear adjustment to an equilibrium configuration. We consider the effect of the vortex initial amplitude and size on its further evolution and analyse the timescales of the adjustment process. In Sect. 4 we discuss the stability and structure of the developed vortex structures. In Sect. 5 we study the process of wave emission induced by the vortex and the subsequent shock development. A study on the effects of numerical resolution is given in Sect. 6 . The paper is finally summarized in Sect. 7.

\section{Numerical code and setup}

We solve the equations of inviscid compressible gas dynamics in two dimensional polar coordinates $(r, \phi)$. From the observer's frame, let $\rho, m_{r}, L_{\phi}$ and $E$ be, respectively, the fluid density, radial, angular momentum and total energy density; their conservation is expressed by

$$
\begin{aligned}
& \frac{\partial \rho}{\partial t}+\frac{1}{r} \frac{\partial\left(r \rho v_{r}\right)}{\partial r}+\frac{1}{r} \frac{\partial\left(\rho v_{\phi}\right)}{\partial \phi}=0, \\
& \frac{\partial\left(\rho v_{r}\right)}{\partial t}+\frac{1}{r} \frac{\partial\left(r \rho v_{r}^{2}\right)}{\partial r}+\frac{1}{r} \frac{\partial\left(\rho v_{r} v_{\phi}\right)}{\partial \phi}+\frac{\partial p}{\partial r}=\frac{\rho v_{\phi}^{2}}{r}+\rho g_{r}, \\
& \frac{\partial L_{\phi}}{\partial t}+\frac{1}{r} \frac{\partial\left(r L_{\phi} v_{r}\right)}{\partial r}+\frac{1}{r} \frac{\partial\left(L_{\phi} v_{\phi}+r p\right)}{\partial \phi}=0, \\
& \frac{\partial E}{\partial t}+\frac{1}{r} \frac{\partial\left[r(E+p) v_{r}\right]}{\partial r}+\frac{1}{r} \frac{\partial\left[(E+p) v_{\phi}\right]}{\partial \phi}=m_{r} g_{r},
\end{aligned}
$$

where $v_{r}$ and $v_{\phi}=L_{\phi} /(\rho r)$ are the radial and azimuthal velocities, $p$ is the thermal pressure and $g_{r}=-1 / r^{2}$ is the gravitational acceleration. The total energy $E$ of the fluid is expressed as the sum of the kinetic and internal energy via the ideal equation of state:

$E=\frac{p}{\Gamma-1}+\rho \frac{v_{r}^{2}+v_{\phi}^{2}}{2}$

where $\Gamma=5 / 3$ is assumed. Dissipative effects are only those related to the use of a mesh of finite width Eqs. (1)-(4) are solved using the hydrodynamics module of the PLUTO code (Mignone et al. 2007). PLUTO evolves the conservative equations in a Godunov-type fashion by first interpolating volume averages inside each cell and then solving a Riemann problem at each interface to compute the fluxes. For the present work, we choose second order slope-limited interpolation and the twostage Runge Kutta time stepping scheme of Gottlieb \& Shu (1998). An accurate Riemann solver based on the two-shock approximation (Colella \& Woodward 1984) is used to compute numerical fluxes.

Integration of the equations is made considerably faster by taking advantage of the FARGO scheme (Masset 2000), available in the PLUTO code. This scheme allows larger timesteps than the standard integration by removing the average azimuthal velocity from the Courant condition, which severely limits the time step because of the fast orbital motion at the inner boundary. Besides the increased computational efficiency, this technique considerably reduces the amount of numerical diffusion in the solution.

Our computational domain covers the region $0 \leq \phi \leq 2 \pi$, $R_{\text {in }} \leq r \leq R_{\text {out }}$, where $R_{\text {in }}$ and $R_{\text {out }}$ are the innermost and outermost boundaries in the radial direction. The grid is constructed by first assigning the number $N_{\phi}$ of uniform zones in the $\phi$ direction, whence $\Delta \phi=2 \pi / N_{\phi}$. The mesh spacing in the radial direction is then chosen to yield approximately equal radial and azimuthal lengths $\Delta r_{i}=r_{i} \Delta \phi$ on each ring $i=1, \ldots, N_{r}$ of the disk. The number of radial zones $N_{r}$ is determined by the condition $r_{i}+\Delta r_{i} / 2=r_{i+1}-\Delta r_{i+1} / 2$ (grid continuity) at each node, giving a first (non-integer) estimate

$N_{r}^{*}=\log \left(\frac{R_{\text {out }}}{R_{\text {in }}}\right) / \log \left(\frac{N_{\phi}+\pi}{N_{\phi}-\pi}\right)$.

Finally, we obtain $N_{r}$ by rounding $N_{r}^{*}$ up to the nearest integer which requires $R_{\text {out }}$ to be properly adjusted by inverting Eq. (6) with $N_{r}^{*}$ replaced by $N_{r}$. Hence, the radial resolution is set up by the azimuthal spacing and by the innermost and outermost boundaries.

The initial condition, at $t=0$, consists of a Keplerian disk with uniform density and pressure plus a velocity perturbation described in the following subsection.

Boundary conditions are set as follows: at the outermost radius free outflow is permitted, whereas at the innermost radius variables are kept constant to their initial value. Periodic boundary conditions hold in the azimuthal domain.

For our simulations we used four main numerical setups, with low, medium, high and very high resolutions (called respectively $\mathrm{L}, \mathrm{M}, \mathrm{H}$ and $\mathrm{VH}$ ), described in detail in Table 1.

\subsection{Initial vortex}

Initial conditions for numerical simulations are composed by the sum of the Keplerian flow with $V_{\phi}=r^{-1 / 2}$ and a vortex perturbation, that can have different geometries, size and amplitude. The 
Table 1. Resolution, radial domain and number of revolutions at $r=1$ for the different numerical setups. The domain length in the angular direction is $\phi \in[0,2 \pi]$.

\begin{tabular}{lccc}
\hline \hline & $\begin{array}{c}\text { Resolution } \\
\left(N_{\phi} \times N_{r}\right)\end{array}$ & $\begin{array}{c}\text { Domain } \\
{\left[R_{\text {in }}, R_{\text {out }}\right]}\end{array}$ & $\begin{array}{c}\text { Time } \\
(\text { Revol. })\end{array}$ \\
\hline L....... & $1500 \times 550$ & {$[0.2,2]$} & 80 \\
M...... & $2000 \times 622$ & {$[0.4,4]$} & 200 \\
H...... & $4000 \times 1466$ & {$[0.2,2]$} & 80 \\
VH...... & $8000 \times 1559$ & {$[0.5,1.7]$} & 80 \\
\hline
\end{tabular}

initial vortex configuration most widely used in our simulations is the following:

$v_{x}^{\prime}(0)= \pm \frac{\epsilon\left(y-y_{0}\right)}{q} \exp \left[-\frac{\left(x-x_{0}\right)^{2}}{a^{2}}-\frac{\left.\left(y-y_{0}\right)^{2}\right)}{q^{2} a^{2}}\right]$,

$v_{y}^{\prime}(0)=\mp \epsilon q\left(x-x_{0}\right) \exp \left[-\frac{\left(x-x_{0}\right)^{2}}{a^{2}}-\frac{\left.\left(y-y_{0}\right)^{2}\right)}{q^{2} a^{2}}\right]$,

$\rho^{\prime}(0)=0$.

Here $\epsilon$ defines the amplitude of the initial perturbation and its sign determines the vortex polarity (positive in the case of anticyclonic vortex). The parameters $a$ and $q$ describe, respectively, the size (in the radial direction) and aspect ratio of an elliptic vortex. A circular vortex corresponds to $q=1$, and $q>1$ refers to a vortex elongated in the azimuthal direction. We choose the location of the vortex $\left(x_{0}, y_{0}\right)$ so that it corresponds to the radial location $r_{0}=1$. With these choices, our unit of length is the radius $r_{0}$ at which the vortex is located and our unit of time is the inverse of the Keplerian angular velocity $\Omega_{\mathrm{Kep}}\left(r_{0}\right)$ at the same location. From a physical point of view, the vortex amplitude $\epsilon$ is related to the maximum vorticity $\Omega_{\mathrm{M}}$ (at the vortex center), $\Omega_{\mathrm{M}}=2 \epsilon$

A second type of initial vortex with algebraic distribution of potential vorticity is also used,

$$
\begin{aligned}
& v_{x}^{\prime}(0)= \pm \frac{\epsilon\left(y-y_{0}\right)}{q}\left(1+\frac{\left(x-x_{0}\right)^{2}}{a^{2}}+\frac{\left.\left(y-y_{0}\right)^{2}\right)}{q^{2} a^{2}}\right)^{-1}, \\
& v_{y}^{\prime}(0)=\mp \epsilon q\left(x-x_{0}\right)\left(1+\frac{\left(x-x_{0}\right)^{2}}{a^{2}}+\frac{\left.\left(y-y_{0}\right)^{2}\right)}{q^{2} a^{2}}\right)^{-1} .
\end{aligned}
$$

This initial vortex is used for the sake of comparison to see how different initial potential vorticity fields with similar amplitude and scale but different geometry behave in the nonlinear regime.

\section{Vortex evolution and nonlinear adjustment}

\subsection{The simulations}

The evolution of the perturbations depends on three main parameters: the amplitude and size of the perturbation (respectively $\epsilon$ and $a$ ) and the sound speed in the disk, $c_{\mathrm{s}}$. Our first aim is to determine the region, in the parameter space described by $\epsilon, a$ and $c_{\mathrm{s}}$, in which the evolution of the initial perturbation leads to a stable, long-lived equilibrium configuration. As we shall see, before reaching this final state the system undergoes, in the course of several disk revolutions, a transition phase that we call nonlinear adjustment. Our second aim is to provide a detailed description of the equilibrium vortex configuration. With these purposes we have performed runs with different values of the
Table 2. Table of parameters used for the computations. From the left to right, the columns give the sound speed $\left(c_{\mathrm{s}}\right)$, the vortex initial size $(a)$, amplitude $(\epsilon)$, ellipticity parameter $q$, geometry (namely the shape of the initial vorticity distribution: exp and alg stand for the initial configurations given by Eqs. (7), (8) and (10), (11), respectively) together with its polarity $\mathrm{Pol}$ ( - for anticyclonic vortices and + for cyclonic ones). The numerical setup (NS, given in Table 1) and an identification label

\begin{tabular}{|c|c|c|c|c|c|c|}
\hline$\overline{c c_{\mathrm{S}}}$ & $\bar{a}$ & $\bar{\epsilon}$ & $\overline{q q}$ & "Geom/Pol & $\overline{\mathrm{NS}}$ & $\overline{\text { Label }}$ \\
\hline \multirow[t]{7}{*}{0.1} & 0.1 & 0.1 & $\frac{1}{1}$ & $\exp /-$ & $\mathrm{L}$ & A1 \\
\hline & & 0.2 & 1 & $\exp /-$ & $\mathrm{L}$ & A2 \\
\hline & & 0.3 & 1 & $\exp /-$ & $\mathrm{L}$ & $\mathrm{A} 3$ \\
\hline & & 0.5 & 1 & $\exp /-$ & $\mathrm{H}$ & A4 \\
\hline & & 0.8 & 1 & $\exp /-$ & $\mathrm{L}$ & A5 \\
\hline & 0.3 & 0.5 & 1 & $\exp /-$ & $\mathrm{L}$ & A6 \\
\hline & 0.4 & 0.5 & 1 & $\exp /-$ & $\mathrm{L}$ & A7 \\
\hline 0.05 & 0.3 & 0.5 & 1 & $\exp /-$ & $\mathrm{M}$ & B1 \\
\hline \multirow[t]{13}{*}{0.01} & 0.01 & 0.5 & 1 & $\exp /-$ & $\overline{\mathrm{VH}}$ & $\mathrm{C} 1$ \\
\hline & & 1. & 1 & $\exp /-$ & $\mathrm{H}$ & $\mathrm{C} 2$ \\
\hline & 0.02 & 0.5 & 1 & $\exp /-$ & $\mathrm{H}$ & $\mathrm{C} 3$ \\
\hline & 0.05 & 0.5 & 1,2 & exp,alg/- & VH & $\mathrm{C} 4$ \\
\hline & 0.1 & 0.1 & 1 & $\exp /-$ & $\mathrm{H}$ & C5 \\
\hline & & 0.2 & 1 & $\exp /-$ & $\mathrm{H}$ & C6 \\
\hline & & 0.22 & 1 & $\exp /-$ & $\mathrm{H}$ & C7 \\
\hline & & 0.25 & 1 & $\exp /-$ & $\mathrm{H}$ & C8 \\
\hline & & 0.28 & 1 & $\exp /-$ & $\mathrm{H}$ & C9 \\
\hline & & 0.3 & 1 & $\exp /-$ & $\mathrm{H}$ & $\mathrm{C} 10$ \\
\hline & & 0.5 & 1 & $\exp /-$ & $\mathrm{H}$ & $\mathrm{C} 11$ \\
\hline & 0.2 & 0.2 & 1 & $\exp /-$ & M & $\mathrm{C} 12$ \\
\hline & & 0.5 & 1 & $\exp /-,+$ & $\mathrm{H}$ & $\mathrm{C} 13$ \\
\hline \multirow[t]{2}{*}{0.005} & 0.02 & 0.5 & 1 & $\exp /-$ & $\mathrm{H}$ & D1 \\
\hline & 0.025 & 0.5 & 1 & $\exp /-$ & $\mathrm{H}$ & D2 \\
\hline 0.002 & 0.02 & 0.5 & 1 & $\exp /-$ & $\mathrm{H}$ & E1 \\
\hline \multirow[t]{4}{*}{0.001} & 0.01 & 0.5 & 1 & $\exp /-$ & $\mathrm{H}$ & E2 \\
\hline & 0.02 & 0.5 & 1 & $\exp /-$ & $\mathrm{H}$ & E3 \\
\hline & 0.05 & 0.5 & 1 & $\exp /-$ & $\mathrm{H}$ & E4 \\
\hline & 0.1 & 0.5 & 1 & $\exp /-$ & $\mathrm{H}$ & E5 \\
\hline
\end{tabular}
are given in the rightmost columns.

three parameters and different numerical setups, using Eqs. (7), (8) with $q=1$ (circular vortices). In particular, by increasing the value of the sound speed $\left(c_{\mathrm{s}}=0.001,0.01,0.1\right)$, we have explored the behavior of the vortex changing $a$ at fixed $\epsilon$ and changing $\epsilon$ at fixed $a$. Additional calculations have been performed for the purpose of exploring in more detail particular regions of the parameter space. For instance, additional values of $c_{\mathrm{S}}$ have been used for a better understanding of the scaling behaviors of some of the vortex properties. Moreover, in order to investigate whether the general behavior is changed by varying the shape and structure of the initial perturbation, we have performed computations with different values of the ellipticity parameter $q$ and of the (algebraic) potential vorticity distribution (see section above). A complete list of all the simulations and related parameters are described in Table 2 .

\subsection{Nonlinear amplitude thresholds}

It is well known that vortices with small amplitudes are sheared away by the linear deformation induced by the background flow. Nonlinear effect can counteract the shearing deformation only for vortices with higher amplitude. In this respect, we found two threshold values for $\epsilon$ defining the character of the evolution of the initially imposed vortex. The first threshold value between linear and nonlinear behaviors is:

$\epsilon^{*}=0.1$. 
For $\epsilon<\epsilon^{*}$ the vortex behaves linearly (Bodo et al. 2005). Its dynamics is characterized by the geometrical stretching of the initial configuration, due to the strong radial Keplerian velocity shear. The linear vortex is then stretched to fill the entire $(2 \pi)$ azimuthal domain, within the first 1.5 local disk revolutions. It then experiences further decay down to the dissipation scales, where it is damped.

When the vortex amplitude exceeds the first nonlinear threshold, a two-stage process occurs. First, the vortex is sheared into a narrow vortex layer, which then undergoes local instabilities. We then observe the formation of small-scale weak anticyclonic vortices at different azimuthal locations. Hence, the initial vortex is transformed into a chain of small scale vortices that tend to spread and occupy the entire azimuthal domain. The typical character of the evolution of potential vorticity for this case is shown in Fig. 1.

Increasing the initial vortex amplitude, we found a second nonlinear threshold:

$\epsilon^{* *}=0.25$.

Vortices with $\epsilon>\epsilon^{* *}$ experience direct adjustment from the initial to the final persistent structure, i.e. a strong anticyclonic vortex. This process may be followed in Fig. 2, where an initially unbalanced vortex, within 4 revolutions, undergoes direct adjustment to the final nonlinear configuration. The energy excess of the initial state is radiated away in the form of spiral-density waves and shocks.

The final equilibrium configuration appears to be a nonlinear attractor reached by the system if the initial amplitude exceeds $\epsilon^{* *}$ and if the initial spatial scale falls in a range discussed in the next subsection. Indeed, the same nonlinear state is developed from all initial vortices satisfying these conditions, independently from the details of the initial potential vorticity distribution (exponential or algebraic, circular or elliptic).

\subsection{Initial vortex size}

The result of nonlinear adjustment strongly depends also on the initial vortex size. In order to quantify this dependence, we have carried out computations with vortices of different initial size $a$. The size of the vortex can be compared with the length-scale

$H \equiv c_{\mathrm{s}} / \Omega_{\mathrm{Kep}}$,

which, in presence of the vertical component of gravity, describes the scale height of a thin Keplerian disk. From a physical point of view, a 2D analysis can give meaningful results only for vortices with sizes larger than $H$. This will be our case of interest. For $c_{\mathrm{s}}$ initially constant all over the disk (as it is in our case), $H$ is a function of the radial position and, at the vortex location, one has $H=c_{\mathrm{s}}\left(\right.$ since $\left.\Omega_{\mathrm{Kep}}(1)=1\right)$.

Our numerical results show that vortices undergoing direct nonlinear adjustment have initial size in a range $a_{0}<a<a_{\max }$, where the limits $a_{0}$ and $a_{\max }$ depend on the local disk parameters. During the nonlinear adjustment the vortex decreases its size, radiating the excess energy through spiral density waves and shocks, and reaches the equilibrium configuration with size $a_{0}$ that can be still larger than the disk scale height. The final equilibrium configuration reached by these vortices appears to be independent from the details of the initial state and seems to represent a nonlinear attractor, whose characteristics will be described in detail in the next section. In cases $\mathrm{C} 1-\mathrm{C} 13$ (i.e., $c_{\mathrm{s}}=10^{-2}$ ), the value range of $a$ evolving into the same nonlinear configuration is $2 H<a<10 H$. For comparison, in cases E2-E5 $\left(c_{\mathrm{s}}=10^{-3}\right)$ this range is $12 \mathrm{H}<a<20 \mathrm{H}$.
When the spatial-scale of the initial vortex exceeds $a_{\max }$, the evolution is quite complex. We observe a radial transfer of potential vorticity in both directions (see Fig. 3), caused by the combined action of shocks (radiated from the initially imposed supersonic vortex) and of flow curvature (inducing Rossby wave variations). The initial radial transfer of potential vorticity is accompanied by a shearing process leading to a decrease of potential vorticity localization and to a subsequent fragmentation. The resulting picture tends to become even more complicated when secondary vortices, formed at different radii and with different sizes, start to interact. As a result, we see multiple vortices with typical sizes equal to or smaller than $H$ leaning to further decay in size and amplitude.

When the size of the vortex is smaller than $a_{0}$ and the initial vortex amplitude exceeds the second nonlinear threshold, we observe nonlinear adjustment to a final configuration with sizes similar to the initial values. In this case, developed vortices typically have sizes smaller than $a_{0}$. However, if the initial amplitude largely exceeds the second nonlinear threshold, we have found that the vortex can increase its size and reach the nonlinear attractor discussed above. We have not studied this case $\left(a<a_{0}\right)$ in detail since the typical size of vortices developed during the adjustment falls around $H$ or smaller (unless the initial amplitude is quite high), indicating the importance of $3 \mathrm{D}$ consideration.

Figure 4 shows a sketch diagram illustrating the character of vortex evolution depending on its initial amplitude $(\epsilon)$ and size $(a)$.

In the following, we will focus our attention on the cases that reach the equilibrium configuration. For them we discuss, in the following subsection, the time-scales of nonlinear adjustment and, in the following Sect. 4, the final equilibrium structure.

\subsection{Time-scales of the adjustment}

The characteristic time-scale needed for radiating the energy excess and reaching the equilibrium configuration strongly depends on the vortex size. As we shall see in the next Section, the equilibrium vortex configuration has a characteristic size that does not depend on the initial size of the vortex. This means that bigger initial vortices need to emit more energy to adjust to the final small-scale configuration. Indeed, we have seen that a different number of revolutions were needed to develop a stable long-lived structure starting from vortices of different size ${ }^{1}$ :
$a=0.05 \rightarrow 3$ revolutions,
$a=0.1 \quad \rightarrow \quad 4$ revolutions
$a=0.2 \quad \rightarrow \quad 6 \quad$ revolutions

(runs C4, C11 and C13). The geometry of the potential vorticity adjustment can be seen in Fig. 6. It matches the patterns observed in previous numerical simulations (see e.g. Godon \& Livio 1999b). However, our high-resolution simulations reveal new features of this process. The adjustments of potential vorticity and density proceed in different ways and on different timescales (see Fig. 6). When the potential vorticity has almost reached its equilibrium configuration, the density distribution still undergoes significant structural variations. Initially it develops a double core vortex configuration that slowly tends to change into a one core stable vortex. For an initial value of $a=0.1$, the potential vorticity adjusts in 4 revolution, while

\footnotetext{
1 Although multiple vortices are developed, here we measure the time for the development of the main vortex.
} 

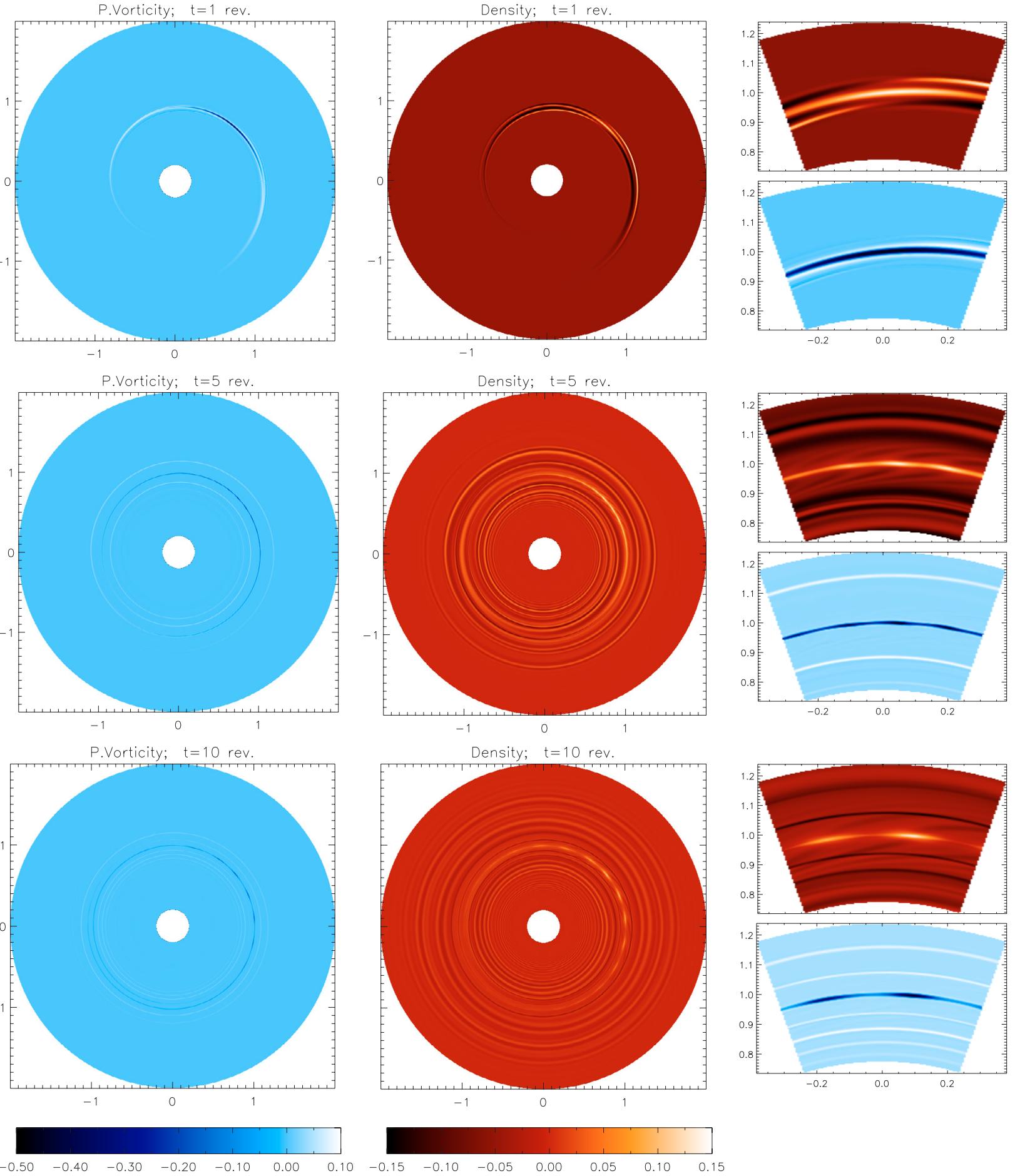

Fig. 1. Dynamics of an initially imposed small-scale anticyclonic vortex (exponential distribution of vorticity, $q=1, a=0.1$ ) with amplitude exceeding the first nonlinear threshold parameter: $\epsilon=0.2$. The system parameters correspond to case C6 (see Table 2). The first column shows the evolution of the perturbed potential vorticity and the second column the evolution of density at times 1,5 and 10 (in units of the local disk revolution period). The third column displays zooms at the vortex location at the same times. The vortical perturbation is initially stretched into a narrow vortex sheet which then undergoes local nonlinear instabilities producing a chain of small-scales anticyclonic vortices that tend to fill the entire azimuthal domain. See electronic version of the paper for color figures.

density needs 15 revolutions for reaching the final configuration (case C11). Moreover, the density adjustment (that is, the time needed for the merging of the two density cores) shows a strong dependence on the intrinsic numerical viscosity. Indeed, increasing the resolution, this settlement time increases. The differences between vorticity and density adjustments may be a consequence of the existence of spiral shocks generated by the interaction of the nonlinear vortex with the background shear flow, as we will discuss in Sect. 5.

\section{Vortex stability and structure}

One of the main goals of the present study is to describe the stability and structure of long-lived vortices in Keplerian disks. For 

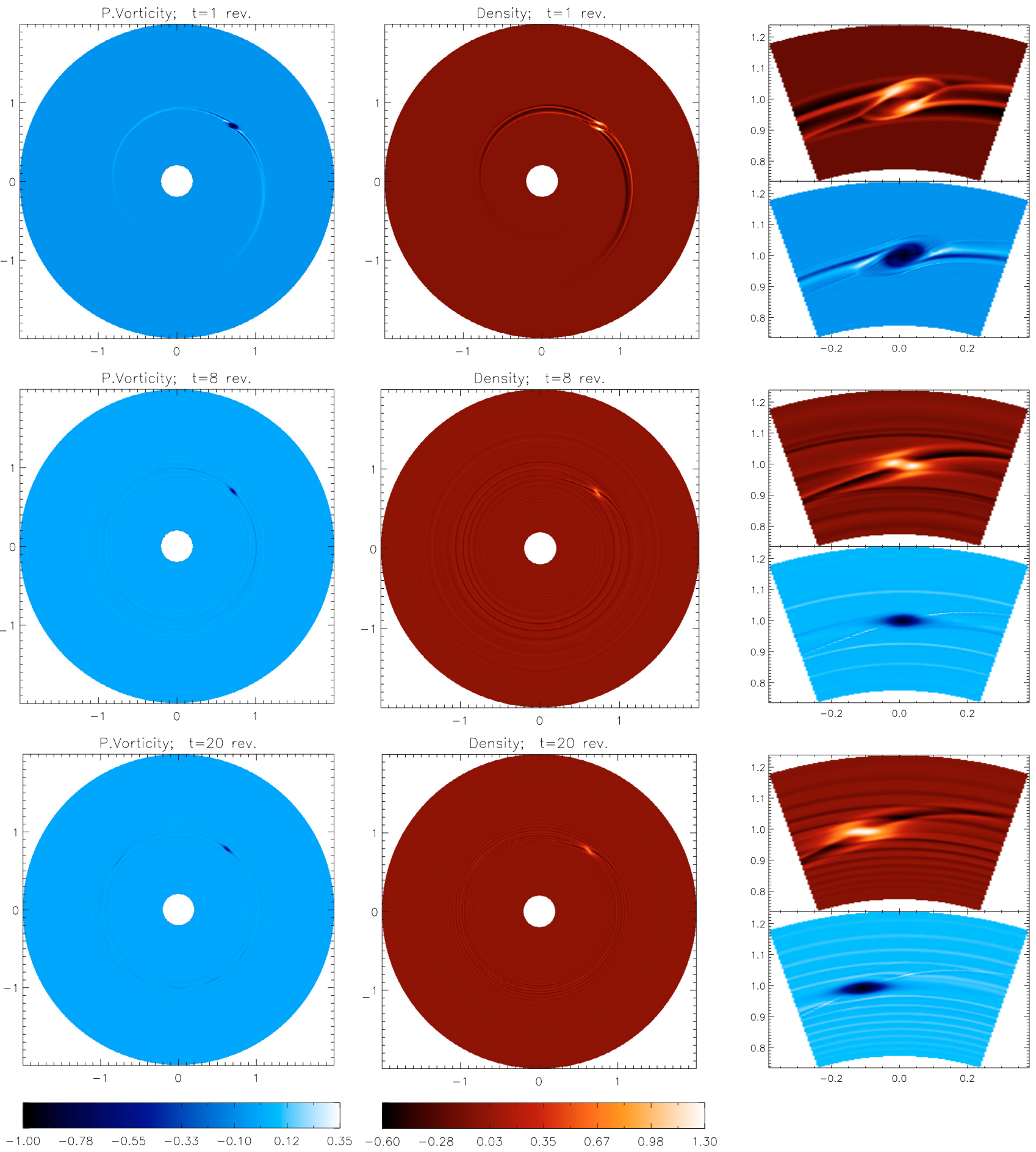

Fig. 2. Dynamics of an initially imposed small-scale anticyclonic vortex (exponential distribution of vorticity, $q=1, a=0.1$ ) with amplitude exceeding the second nonlinear threshold parameter: $\epsilon=0.5$. The system parameters correspond to case C11 (see Table 2). The first column shows the evolution of the perturbed potential vorticity and the second column the evolution of density at times 1,8 and 20 revolutions (in units of the local disk revolution period). The initially imposed vortex with high amplitude emits the excess energy in form of density-spiral waves and within 4 revolutions adjusts to the single nonlinear configuration which survives further without significant variation throughout the entire period of simulation. See electronic version of the paper for color figures.

this purpose, we selected cases undergoing direct adjustment to a single vortex, and we followed their long time behavior. Figure 7 shows radial profiles of potential vorticity and density at the center of a vortex for case C11, integrated for a total of 200 revolutions. After 4-6 rotations the potential vorticity configuration shows a well defined center. The density maximum matches the vorticity center after approximately 15 revolutions. We observe a profound persistent structure throughout 200 revolutions. Of particular interest is the behavior of the mass accumulated by the anticyclonic velocity circulation, shown in Fig. 7, where we 

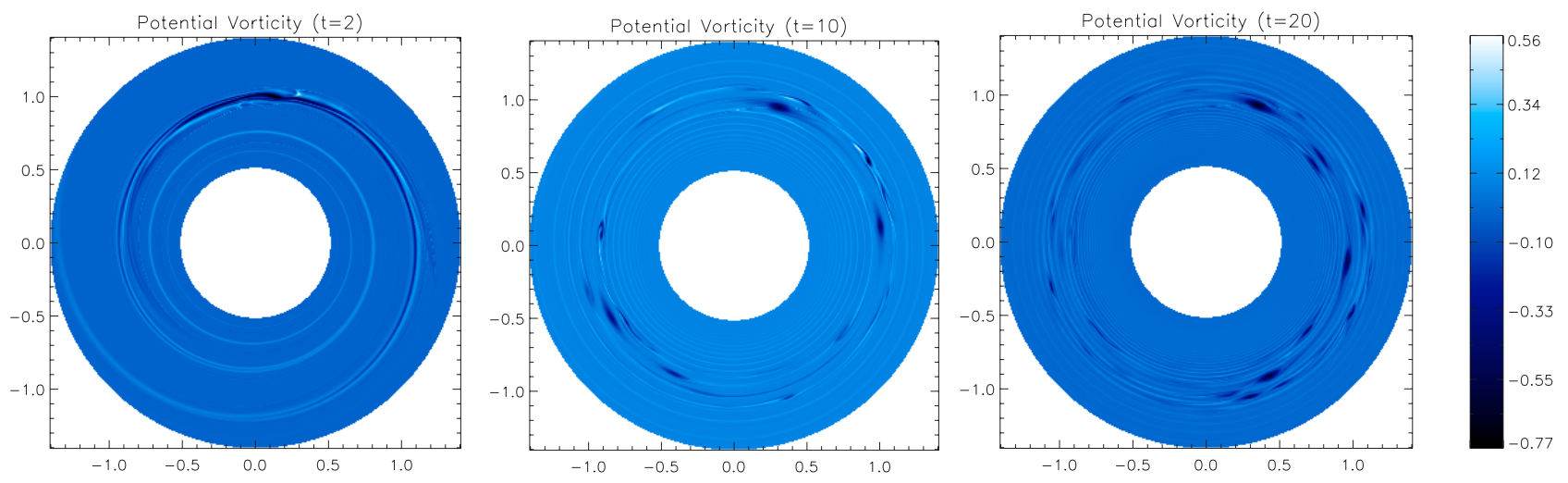

Fig. 3. Potential vorticity evolution in the case of an anticyclonic vortex with exponential distribution of vorticity, $q=1, a=0.2$ and amplitude exceeding the second nonlinear threshold parameter: $\epsilon=0.5$. The system parameters correspond to case C13 (see Table 2). The background Keplerian vorticity is subtracted. The dark spots reveal the anticyclonic patches. The radial transport of potential vorticity occurs on the initial stages, when the vortex is oversized and is subject to curvature radiation. At later times the background shearing stretches the vorticity and developed vortices only move azimuthally, while occasionally interacting and decaying.

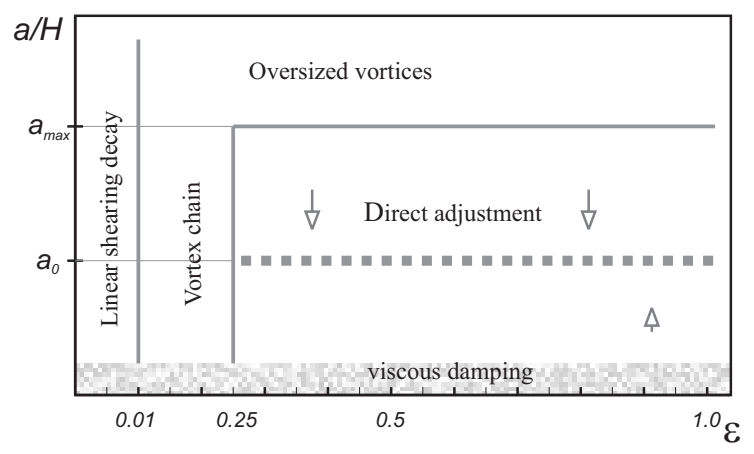

Fig. 4. Sketch diagram illustrating the character of the evolution of an initially imposed anticyclonic vortex. In abscissa we have the amplitude $(\epsilon)$ and in ordinate the size $(a)$ of the initial anticyclonic vortex. Two nonlinear thresholds in amplitude $(0.01$ and 0.25$)$ control the fate of the initial vortex, as it can decay linearly, evolve into a vortex chain or undergo direct adjustment to a single persistent configuration. The initial vortex size determines if the vortex will decay due to radial vorticity transport (oversized vortices), viscosity, or undergo direct adjustment. Horizontal dotted line shows the size of the equilibrium persistent configuration which can be interpreted as the nonlinear attractor.

observe a slow but steady increase of density at the vortex center. The same figure shows the steepening of the vorticity gradient during the adjustment process.

By analyzing vortices with different initial configurations, we have found that the shape of the developed, persistent vortex depends only on the disk sound speed $c_{\mathrm{s}}$, and not on the characteristics of the initial vortex (provided its size $a$ and amplitude $\epsilon$ fall in the ranges discussed in the previous section). We can characterize the final state by its size $a_{0}$ (in the radial direction) and ellipticity parameter $q$. The vortex size is measured defining as vortex the region that has potential vorticity larger than $10 \%$ of the maximum value at the vortex center. The following equation gives a scaling law for $a_{0}$ as function of the sound speed $c_{\mathrm{s}}$ (see Barranco \& Marcus 2005):

$a_{0}=f\left(c_{\mathrm{s}}\right) H$,

where the function $f\left(c_{\mathrm{s}}\right)$ (i.e. the ratio $\left.a_{0} / H\right)$ is shown in Fig. 5. Note, that although our vortex scales with the sound speed (and the disk height scale), we have found vortices with maximal size larger than the ones described in Barranco \& Marcus (2005). The plot shows that the ratio $a_{0} / H$ increases for decreasing the

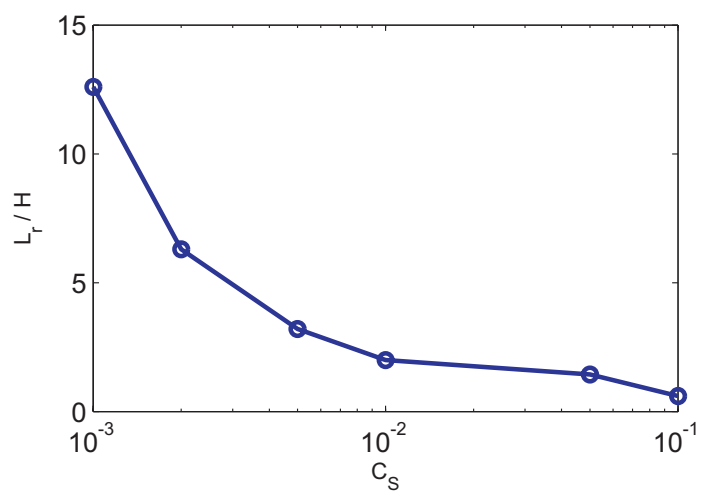

Fig. 5. Scaling law for the equilibrium vortex size: $f\left(c_{\mathrm{s}}\right)\left(=a_{0} / H\right)$ as a function of sound speed $c_{\mathrm{s}}$. The radial extent of the vortex is used.

sound speed, so the condition for a 2D vortex behavior (vortex size larger than the disk scale height), is verified to a better extent at lower sound speeds. The final ellipticity $q$, on the other hand, appears to be almost independent from $c_{\mathrm{s}}$ and has a typical value $\sim 5$.

We confirm that the initial vortex configuration, for giving direct adjustment, has to satisfy the condition derived in Davis et al. (2000): the velocity gradient in the center of the initial vortical field must exceed the Keplerian gradient. Moreover, we observe that the velocity gradient of the perturbation tends to decrease during its evolution and reaches the Keplerian value in the equilibrium configuration.

Figure 9 shows the decay curves of the maximum of potential vorticity at the center of the vortex for different resolutions. As expected, for finer meshes, the vortex decay is slower because of the reduced numerical dissipation. However, apart from this consideration, interesting effects can be noticed. After a rapid variation within the adjustment time (first 5 revolutions in present case), one can observe the formation of a stable persistent vortex. Interestingly, the vortex seems to be temporarily able to oppose viscous dissipation, exhibiting for some time an increase of the maximum potential vorticity. This effect implies production of potential vorticity. On the other hand, potential vorticity is a nonlinearly conserved quantity in barotropic flows. Thus, the source of the coherent generation of potential vorticity necessary to support or even enhance the vortex are the spiral 


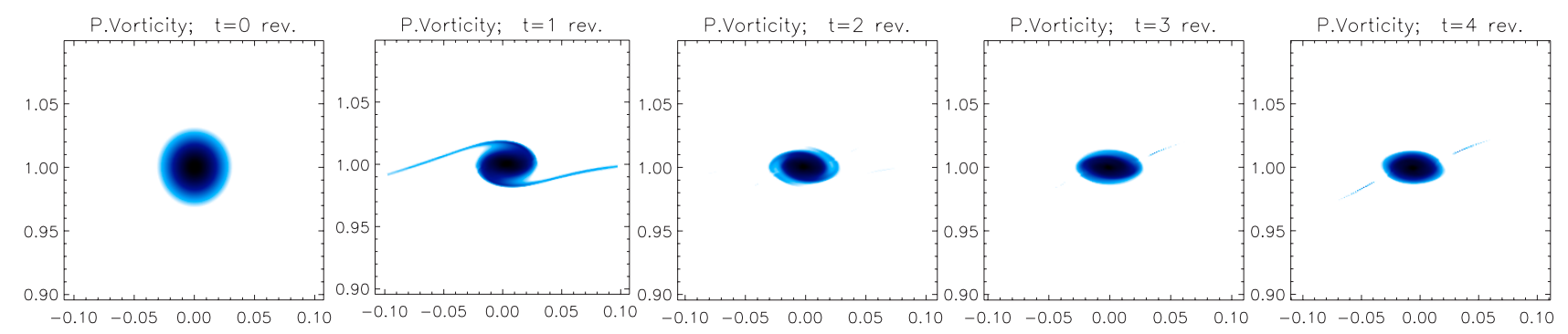

Fig. 6. Evolution of potential vorticity during the direct nonlinear adjustment. The initial conditions correspond to a small-scale anticyclonic vortex with $\epsilon=0.5, a=0.05$ and $q=1$. The system parameters correspond to case C4 (see Table 2). Potential vorticity of the initially imposed vortical perturbations undergoes adjustment to the final equilibrium state within the first 3 revolutions.
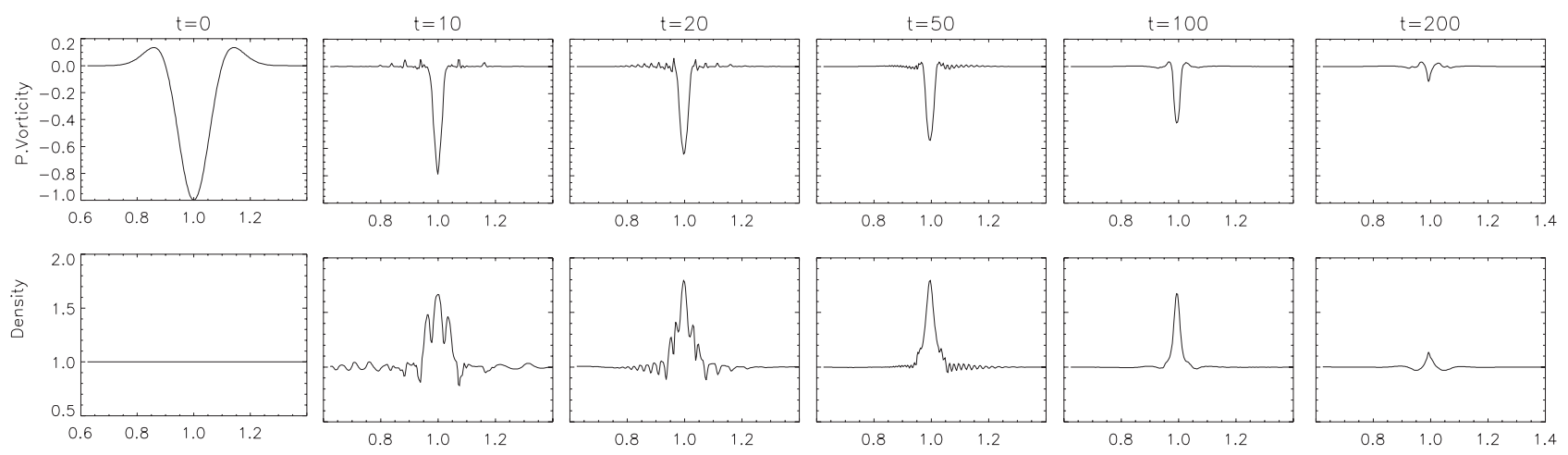

Fig. 7. Evolution of potential vorticity and density for the anticyclonic vortex with $\epsilon=0.5, a=0.1, q=1$ (setup C11). Radial cuts are taken across the geometrical center of the vortex (corresponding to a minimum in potential vorticity, for anticyclonic vortices). The figures show a gradual increase of density in the vortex core. Viscous forces damp oscillations around the vortex. At higher resolution this effect occurs on longer time-scales. After the initial adjustment, the vortex persists in the flow for long times and its dynamics is only a subject to viscous damping.

shock waves that will be discussed in Sect. 5. They seem to be able to overcome the (numerical) dissipation at early times, but not at later times. The situation is quite complex, one of the possible reasons for this behavior could be a change in the internal vortex structure, like the transition from the double to the single density core.

\subsection{Cyclonic vortices}

The nonlinear dynamics of cyclonic vortices shows significant differences from anticyclonic ones. In the limit of small amplitudes, cyclonic vortices decay on the shearing timescale, related to the geometrical stretching induced by the background differential rotation. For higher amplitudes (exceeding the first nonlinear threshold $\epsilon^{*}$ ) they exhibit a rapid decay on time-scales shorter than the shearing time-scales. It then appears that nonlinear forces accelerate the linear decay of cyclonic vortices.

\section{Waves and shocks}

Vortices in shear flows generate spiral density waves by a linear mechanism first described by Chagelishvili et al. (1997), and further investigated numerically in Keplerian flows by Bodo et al. (2005). In the present context we study the characteristics of this process when nonlinear forces are also in action. As clearly seen from Figs. 1, 2 and 6, the dynamics of potential vorticity is accompanied by wave emission, not only during the transition time, but also afterwards, when the nonlinear self-sustained vortex is fully developed. This enforce the fact that nonlinearity does not suppress the wave emission. Moreover, after the adjustment, waves emitted by the coherent vortex structure appear to develop into spiral shocks.

A regular structure of spiral shock waves has been found in protoplanetary disk simulations with an embedded protoplanet (Tanigawa \& Watanabe 2002; Koller et al. 2003). Traces of shock generation by vortices may be found in Johnson \& Gammie (2005). In our simulations we verify the existence of a steady pattern of spiral shocks produced by a single vortex. Moreover, shock waves appear to be an inherent property of vortices in sheared compressible flows, but they can be observed only at fairly high resolutions using shock capturing schemes (limits on resolution will be discussed in Sect. 6). Our high resolution calculations allow to study these shock waves in great detail. In Fig. 8 we show the distributions of potential vorticity, density, temperature and local Mach number for the vortex and the attached shock waves. One can distinctly recognize a wave-crest of the density-spiral wave developing into a double shock configuration, with the shock ahead (behind) of the vortex facing the outward (inward) region. A couple of much weaker shocks, parallel to the strong ones, appear to be present although they remain barely visible. These shocks strongly affect the density structure of the developed vortex configuration, resulting in a splitting of the vortex core. Eventually, however, the shearing background leads to the merging of the cores, but the shocks persist.

Spiral shocks induced by a wake of planets are believed, in some situations, to be responsible for planet migration (see Papaloizou \& Terquem 2006, and references therein). In our computations no radial variation of the vortex position has been observed.

As seen in previous studies, spiral shocks affect dust accretion rates on the vortex core and thus promote the formation of 

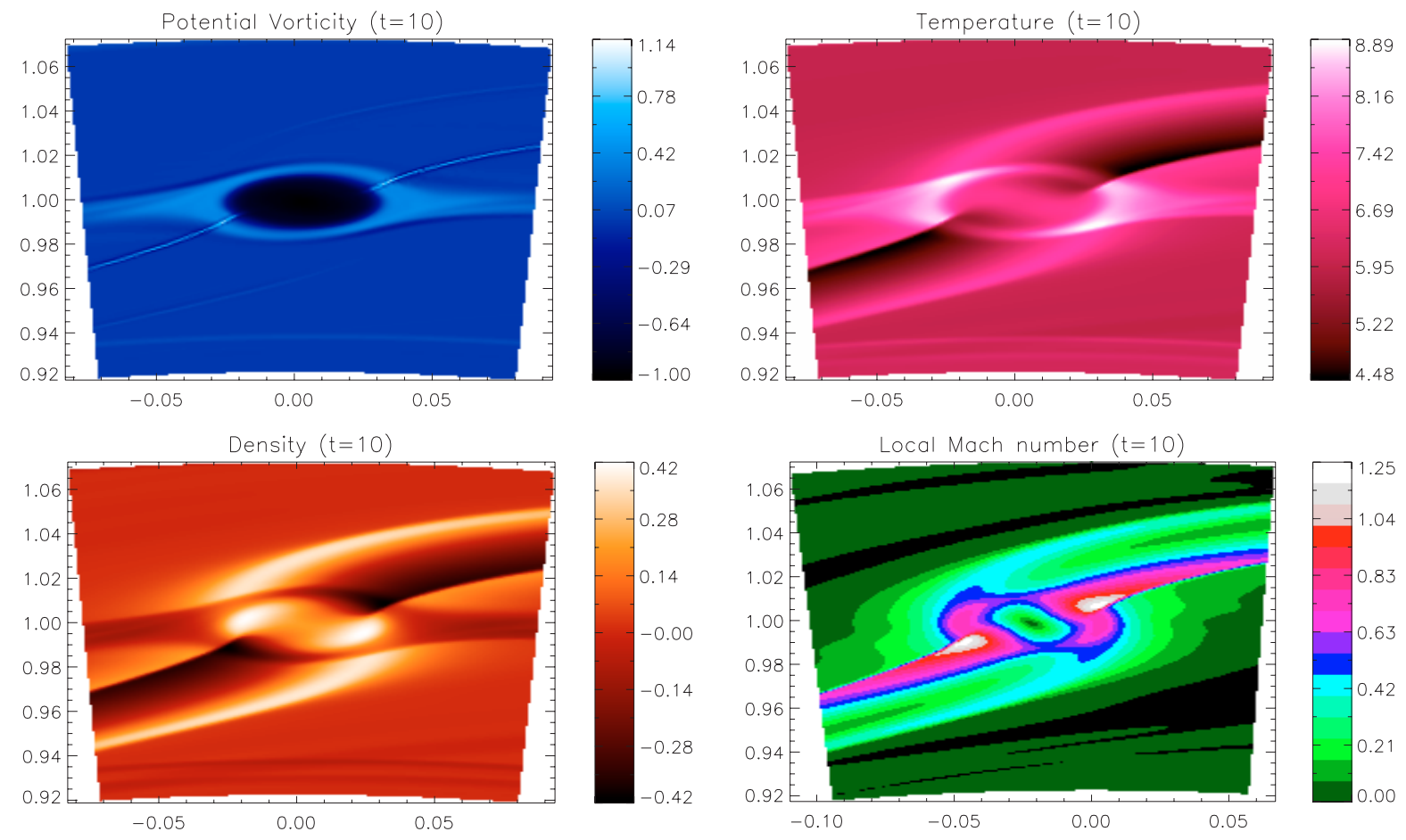

Fig. 8. Potential vorticity, density, temperature and local Mach number of the anticyclonic vortex after 10 revolutions in the disk. Here the initial vortex has $\epsilon=0.5, a=0.05, q=1$ (case C4). Narrow spiral rays beaming out of the central vortex in the potential vorticity panel reveal spiral shocks attached to the outer radius of the vortex. Density perturbations show that compression induced by these shocks are responsible for the double core appearance of the vortex. The temperature plot shows the heating induced by shock dissipation. Heating is stronger in the vicinity of the vortex leading to a consistent temperature increase of the global azimuthal ring that bears the vortex. See electronic version of the paper for color figures.

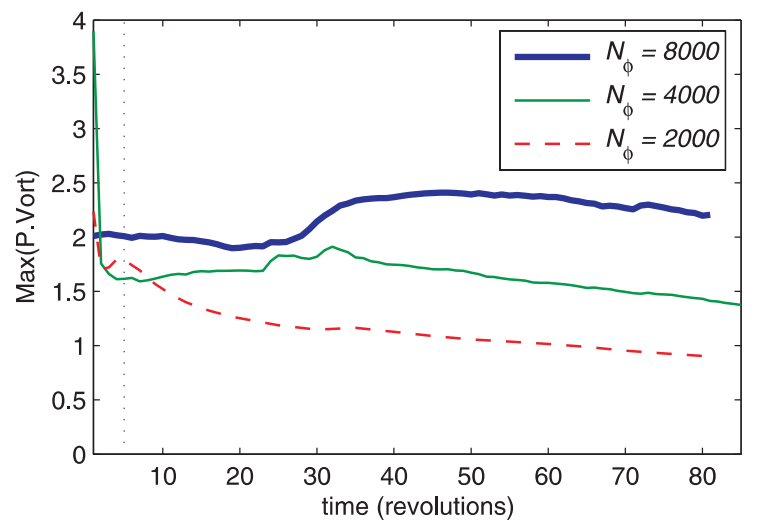

Fig. 9. Plots of the maximum value of potential vorticity (st the vortex center) vs time, at three different resolutions. After the initial adjustment (vertical dotted line) vortex opposes viscous dissipation for some period and falls afterwards into the almost linear decay regime. At 2000 points in the azimuthal direction ( 12 grid points per vortex scale) vortex decays in about the 200 revolutions, while at 4000 point resolution the estimate of the vortex disappearance is at 350 revolutions. At 8000 points the lifespan of the vortex is longer, but can not be confidently estimated using our data.

planetesimal. In this sense, they increase the importance of anticyclonic vortices in planetary formation.

The presence of shocks has consequences for the vortex evolution. Nevertheless, the final fate of these structures cannot be easily foreseen and requires much longer simulations. We can here only sketch some possible scenarios. One possibility is the exhaustion of matter in the vortex bearing ring and the formation of isolated planetesimal. Schäfer et al. (2004) have argued that spiral shocks may lead to the gap formation. On the other hand, shocks heat the ring at the radius where the vortex is sustained (see Fig. 8), which in turn may trigger the linear Rossby wave instability due to the unusual entropy gradient in the disk matter (Lovelace et al. 1999). The instability will induce radial mixing and possibly the destruction of the coherent vortices. On the other hand, Lubow \& Ogilvie (1998) have shown that spiral shocks can be themselves unstable in three dimensions. Hence, as we said, longer and possibly three-dimensional simulations can clarify this issue.

\section{Resolution study and numerical requirements}

Our direct numerical experience shows that a number of stringent numerical requirements may be indispensable for observing the correct dynamics of small-scale vortices in two-dimensional compressible Keplerian flows. One necessary requirement is the need to resolve small size perturbations.

Vortices resolved on 8 computational zones are persistent and, although affected by enhanced numerical viscosity and damping, still survived more than 20 revolutions. Otherwise, at least 24 grid points are necessary to correctly follow the nonlinear adjustment. In Fig. 10 we show an example of such a situation, when numerical setup $\mathrm{M}(2000 \times 733,6$ grid points over vortex) failed to describe the direct nonlinear adjustment of the initially imposed small-scale vortex, but displayed development of a dipolar or even tripolar vortex configurations. Direct nonlinear adjustment to the single long-lived structure is resolved only under considerably higher resolution (setup VH, $8000 \times 1559$, 24 grid points over the vortex) thus setting overall restriction on the resolution of our code. 


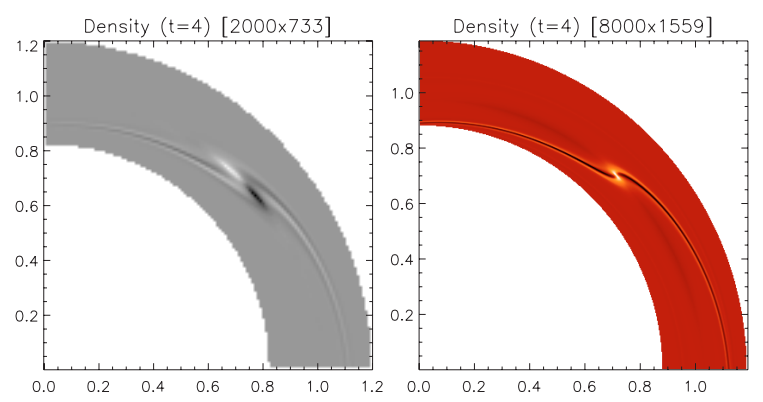

Fig. 10. Dynamics of an initially imposed small-scale anticyclonic vortex with $q=1, a=0.01$ and $\epsilon=0.5$ at two different resolutions: $(2000 \times 733,6$ grid points over the vortex $)$ on the left and $(8000 \times 1559$, 24 grid points) on the right. The fate of the vortex adjustment strongly depends on the resolution. At lower resolution we see the development of a bipolar vortex. Fully resolved vortex development needed resolution as high as 8000 points covering global azimuthal domain.

Notwithstanding the fact that the purpose of the simulations is the study of small-scale vortices, global simulations are needed: vortices interact globally and change the density, temperature and potential vorticity in the whole ring at the radius where they are situated. Hence, to get a correct dynamical picture, we need true global simulations with a domain covering $2 \pi$ in azimuthal direction. Calculations on smaller domains in $\phi(\pi / 2)$ have lead to drastically different results, since the two vortices at the same radius start to interact before the nonlinear adjustment occurs.

Another requirement on the numerical code is that it should be able to capture shocks, an important ingredient of the overall balance of the sustained structures in differentially rotating flows. In our case the PLUTO code employs solvers that accurately resolve the shock dynamics. This requirement does not play in favor of spectral codes for compressible simulations of the Keplerian disk dynamics.

\section{Summary}

Direct numerical simulations of the nonlinear dynamics of coherent vortices in 2D compressible protoplanetary disks with Keplerian differential rotation have been presented.

We have shown the possible existence of anticyclonic vortices with sizes exceeding the Keplerian disk height scales. We have followed the evolution of such vortices for 200 local revolutions, showing their persistence and stability.

We have found that the development of long-lived nonlinear anticyclonic vortex configurations occurs only when the amplitudes of the initially imposed vortex perturbations exceed some threshold value. We have distinguished two nonlinear threshold values, that define the fate of the initial vortex. At small amplitudes, the vortex behaves linearly and it decays due to the shearing deformation. When the amplitude exceeds the first threshold, the vortex stretching is followed by nonlinear instabilities, leading to the formation of multiple small-scale anticyclonic vortices. At amplitudes higher then the second threshold, direct transition from the initially unbalanced to the final equilibrium configuration occurs. We have interpreted the latter process as the nonlinear vortex adjustment and studied the parameters that can describe this process. We found that the vortex adjustment proceeds on two different time-scales. Shorter times are required for the settling the potential vorticity (3-6 revolutions) and longer time-scales are required for the adjustment of density.
Density adjustment time-scales depend on the amount of viscosity present in the flow. Thus, we observe a transient double-core vortex, that evolves into a single core structure. The density core splitting of the vortex is due to the spiral shock induced compression. Cores tend to merge at later times and the process is promoted by the intrinsic numerical viscosity. At lower viscosity, double core vortices survive for longer times. The density at the center of the vortex slowly increases and the potential vorticity gradient steepens.

The structure of the developed long-lived vortex does not depends on the initial vortex configuration, provided it exceeds the second threshold amplitude and its size does not exceed a limiting value. In this sense we found a nonlinear attractor that is the final configuration of a wide range of initial vortical perturbations. We found a scaling law for the equilibrium vortex size. In particular we find that the ratio of the vortex size to the local disk scale height increases with the decrease of the sound speed. Therefore, at low sound speed the radial extent of equilibrium anticyclonic vortices can significantly exceed the disk thickness. In this case vortex dynamics is intrinsically $2 \mathrm{D}$ and should be well modelled by the present simulations. Note that the sound speed required is somewhat lower than the typical values expected for protoplanetary disks $\left(c_{\mathrm{s}} \sim 0.05-0.1\right)$. In the expected range the radial extent would be of the order of the scale height, while the azimuthal extent will be five times larger, in this case, to have a conclusive answer, 3D calculations are needed.

Vortices generate density-spiral waves that rapidly develop into shocks. As a result, a long-lived nonlinearly balanced vortex is accompanied by two spiral compressible shock waves facing both radial directions. The shock strength is maximal in the vicinity of the vortex outer edge, where the shock is attached to the main vortex circulation and heats all the annular disk region containing the vortex. Higher resolution runs indicate that these shock waves are able to generate local potential vorticity and may support the vortex against viscous dissipation.

We analyzed cyclonic vortices at nonlinear amplitudes. It seems that the linear decay due to the shearing deformation is accelerated by nonlinear effects.

We performed a resolution study and found the necessity of a global high-resolution approach to numerical simulations of these processes. The fate of the vortex depends significantly on the dissipation properties of the code. So, in this sense the use of physical viscosity, as opposed to the intrinsic numerical viscosity present in our simulations, would improve understanding of the vortex behavior and damping.

Our study contributes to the scenario of planetary formation inside the core of the long-lived vortices. We found that protoplanetary disks with lower sound speed can sustain vortices with higher ratio of vortex size to disk thickness and create a more favorable conditions for dust trapping and mass accumulation. In this context, we have also found a steady increase of density inside the nonlinearly balanced vortex, partly, due to the existence of persistent, steady, spiral shock waves that we showed to be an intrinsic property of stationary vortices with sizes exceeding the half thickness of the disk.

Acknowledgements. This work is supported by ISTC grant G-1217 and by italian MIUR. A.G.T. would like to acknowledge the hospitality of Osservatorio Astronomico di Torino. Numerical calculations have been partly performed in CINECA (Bologna, Italy) thanks to the support by INAF. 


\section{References}

Adams, F. C., \& Watkins, R. 1995, ApJ, 451, 314

Barge, P., \& Sommeria, J. 1995, A\&A, 295, L1

Barranco, J. A., \& Marcus, P. S. 2005, ApJ, 623, 1157

Bodo, G., Chagelishvili, G., Murante, G., et al. 2005, A\&A, 437, 9

Bracco, A., Chavanis, P. H., Provenzale, A., \& Spiegel, E. A. 1999, Phys. Fluids, 11,2280

Chagelishvili, G. D., Tevzadze, A. G., Bodo, G., \& Moiseev, S. S. 1997, Phys. Rev. Lett., 79, 3178

Chavanis, P. H. 2000, A\&A, 356, 1089

Colella, P., \& Woodward, P. R. 1984, J. Comput. Phys., 54, 174

Davis, S. S. 2002, ApJ, 576, 450

Davis, S. S., Sheehan, D. P., \& Cuzzi, J. N. 2000, ApJ, 545, 494

de la Fuente Marcos, C., \& Barge, P. 2001, MNRAS, 323, 601

Godon, P., \& Livio, M. 1999a, ApJ, 521, 319

Godon, P., \& Livio, M. 1999b, ApJ, 523, 350

Gottlieb, S., \& Shu, C. W. 1998, Math. Comput., 67, 73
Johansen, A., Andersen, A. C., \& Brandenburg, A. 2004, A\&A, 417, 361

Johnson, B. M., \& Gammie, C. F. 2005, ApJ, 635, 149

Klahr, H. H. 2004, ApJ, 606, 1070

Klahr, H. H., \& Bodenheimer, P. 2003, ApJ, 582, 869

Klahr, H. H., \& Bodenheimer, P. 2006, ApJ, 639, 432

Koller, J., Li, H., \& Lin, D. N. C. 2003, ApJ, 596, L91

Lovelace, R. V. E., Li, H., Colgate, S. A., \& Nelson, A. F. 1999, ApJ, 513, 805

Lubow, S. H., \& Ogilvie, G. I. 1998, ApJ, 504, 983

Masset, F. 2000, A\&AS, 141, 165

Mignone, A., Bodo, G., Massaglia, S., et al. 2007, ApJS, 170, 228

Papaloizou, J. C. B., \& Terquem, C. 2006, Rep. Prog. Phys., 69, 119

Petersen, M. P., Stewart, G. R., \& Julien, K. 2007, ApJ, 658, 1252

Schäfer, C., Speith, R., Günther, R., \& Kley, W. 2004, Astron. Nachr. Suppl., 325,85

Shen, Y., Stone, J. M., \& Gardiner, T. A. 2006, ApJ, 653, 513

Tanigawa, T., \& Watanabe, S.-I. 2002, ApJ, 580, 506

Umurhan, O. M., \& Regev, O. 2004, A\&A, 427, 855

von Weiszacker, C. F. 1944, Z. Astrophys., 22, 319 